\title{
Study on Structured-light Stereo Vision Measurement Method under Low-light Environment
}

\author{
Yuanming Ding, Yongchao Zhang, Bin Guo, Weidong Xu \\ Measurement Technology Research and Development Center, \\ Zhejiang Province Institute of Metrology, \\ Hangzhou, 310013, China
}

\begin{abstract}
A structured light binocular stereo vision measurement method was proposed which can be applied to deal with the odometry problem of moving objects in low-light environments such as underwater and enclosed spaces. On the basis of binocular stereo vision system, we use structured light to irradiate the target object. Both the brightness of the object and the feature points' information are increased. The accuracy of feature points' matching is improved in this way. Meanwhile, image correction is used to eliminate the system lens distortion and three-dimensional visual field reduction is achieved based on the matching method of absolute related deviation sum. The experiments' results show that it can measure the distance of the target object and the error is less than $1 \%$ which can meet the requirements in application like underwater odometry and topography mapping. Also image noise reduction and edge detection need to be further studied.
\end{abstract}

Keywords-stereo vision; structured light; low light; measurement; absolute related deviation sum.

\section{INTRODUCTION}

Stereo vision measurement is a non-contact relatively measurement method which is convenient, reliable and high accuracy. It is suitable for industrial inspection[1][2], topography mapping, remote monitoring and other fields, the key technology is three-dimensional imaging, such as laser scanning[3], binocular vision[4], structured light imaging, TOF (Time Of Flight) and so on.

In three-dimensional imaging technology, Lang Haitao[5], who used coded aperture imaging principle to realize three-dimensional imaging, mainly used in measurements such as long-distance and large-scale target of space remote sensing. Huang Hongqiang[6] et al presented a three-dimensional imaging technique based on color-coded structured light which used color as a load and transfer tool. Three-dimensional image information can be gained through analysis and decoding of the color information. Wei Jinsong[7] et al used single-slit streak tube as the receiver of laser three-dimensional imaging which possessed large view field, high frame rate and other characteristics. But it was only applied to long-range target imaging. Cao Sheng[8] et al designed a binocular stereo vision imaging system based on flatbed scanner. Colored stripe can be got by prism and the red, green and blue color filters after the light in spectroscope through optical lens.
The camera can implement three-dimensional imaging by shooting colored stripe.

In this paper a structured light binocular stereo vision measurement method is proposed which can be applied to deal with the odometry problem of moving objects in lowlight environments such as underwater and enclosed spaces. On the basis of binocular stereo vision system, we use structured light to irradiate the target object. The brightness of the object is increased. Also the feature points' information is improved because of linear feature of structured light so as to avoid false matching due to insufficient light. The accuracy of feature points' matching is improved in this way. Meanwhile, image correction is used to eliminate the system lens distortion and threedimensional visual field reduction is achieved based on the matching method of absolute related deviation sum. In experiments, the center of the binocular vision system is set as the coordinate origin. The three-dimensional coordinates of the measured object feature points in view field are extracted and then calculate the distance of the measured object. The experiments' results show that the error is less than $1 \%$ which can meet the requirements in application like underwater odometry and topography mapping.

\section{MEASURING PRINCIPLE BASED ON BINOCULAR VISION}

The Stereo vision measurement system consists of cameras, structured light, the target object and computer. Through casting bunch of strip light on the target object by the structured light and then the left and right cameras do stereo matching according to the characteristics of the target object. Therefore the position relationship of the target object and measuring system can be obtained. Figure 1 shows the imaging principle.

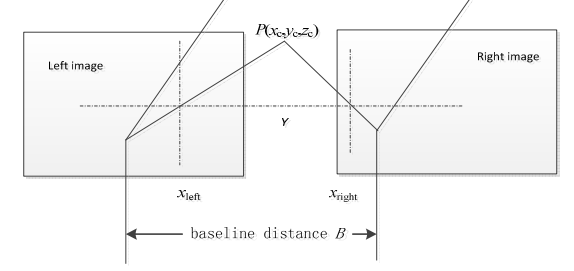

Figure 1. The measuring principle of the stereo vision

The two cameras are placed in parallel in this paper which ensures the same $\mathrm{Y}$-axis. The baseline distance $B$ 
represents the distance between the optical center of the lens of two cameras and the focal length is $f$. The origin of camera coordinate system is coincident with the optical center of the left camera. $P_{\text {right }}\left(x_{\text {right }}, y_{\text {right }}\right)$ and $P_{\text {left }}\left(x_{\text {left }}, y_{\text {left }}\right)$ are the images in the two cameras of point $P\left(\mathrm{x}_{\mathrm{c}}, \mathrm{y}_{\mathrm{c}}, \mathrm{z}_{\mathrm{c}}\right)$ in space. The ordinate $y_{\text {left }}$ and $y_{\text {right }}$ are equal to $y$ since the two cameras are on the same level. Figure 2 shows the image of the point in plane XZ.

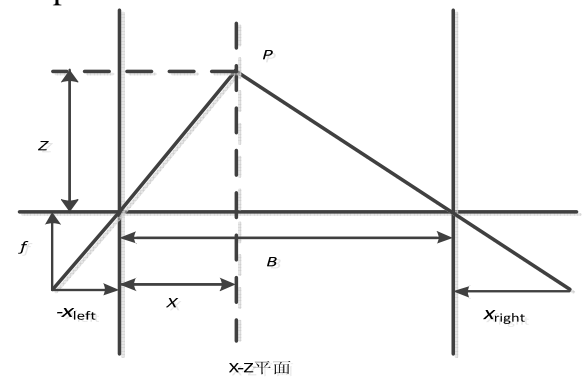

Figure 2. The plane of $\mathrm{X}-\mathrm{Z}$

Wherein $\mathrm{Z}$ represents the distance from the camera to the target position. B represents the baseline distance. $f$ represents the focal length. $X$ is the coordinate value of the point $p$ in the world coordinate' $\mathrm{X}$ axis direction. The coordinates in two cameras' image plane are $x_{\text {left }}$ and $x_{\text {right }}$.

The following relationships hold according to the principle of similar triangles.

$$
\left\{\begin{array}{l}
x_{\text {left }}=\frac{f}{Z} X \\
y=\frac{f}{Z} Y \\
x_{\text {right }}=-\frac{f}{Z}(B-X)
\end{array}\right.
$$

$\mathrm{XYZ}$ represents the position of the target points in the world coordinate system. The formula 2 can be derived from formula 1 so as to get the world coordinates of the target points.

$$
\left\{\begin{array}{l}
X=\frac{x_{\text {left }}}{X_{\text {left }}-X_{\text {right }}} B \\
Y=\frac{y}{x_{\text {left }}-x_{\text {right }}} B \\
Z=\frac{f}{x_{\text {left }}-x_{\text {right }}} B
\end{array}\right.
$$

The formula 3 represents the converted relationship between the pixel coordinate system and the image coordinate system.

$$
\left[\begin{array}{l}
u \\
v \\
1
\end{array}\right]=\left[\begin{array}{ccc}
\frac{1}{d x} & 0 & u_{0} \\
0 & \frac{1}{d y} & v_{0} \\
0 & 0 & 1
\end{array}\right] \times\left[\begin{array}{l}
x \\
y \\
1
\end{array}\right]
$$

$(u, v)$ represents the corresponding points' pixel coordinates. $\left(u_{0}, v_{0}\right)$ represents the center of the pixel coordinate. $(x, y)$ represents the corresponding points' coordinates in the image coordinate. $\left(d_{\mathrm{x}}, d_{\mathrm{y}}\right)$ represents physical size of a pixel. The model of binocular parallel stereo vision system is transformed which can be described via the formula 4 by taking formula 3 and formula 2 .

$$
\left\{\begin{array}{l}
X=\frac{u_{\text {left }}-u_{0}}{u_{\text {left }}-u_{\text {right }}} B \\
Y=\frac{\left(v_{\text {left }}-v_{0}\right) d y}{\left(u_{\text {left }}-u_{\text {right }}\right) d x} B \\
Z=\frac{f B}{\left(u_{\text {left }}-u_{\text {right }}\right) d x}
\end{array}\right.
$$

$\left(u_{\text {right }}, v_{\text {right }}\right)$ and $\left(u_{\text {left }}, v_{\text {left }}\right)$ denote the corresponding point' coordinates value of the pixel coordinates in two cameras. The key to determine the relative position between the object and measurement system is the extraction of left and right cameras' corresponding point. Extraction and matching of feature points are the major measures to get the corresponding points. The information of feature points can be increased by using structured light in low-light environment which so as to facilitate the extraction of feature points. The method of feature points matching is based on absolute related deviation sum and the matching evaluation function is shown in Formula 5.

$$
J=\sum_{i=-\frac{m}{2}}^{\frac{m}{2}} \sum_{j=-\frac{m}{2}}^{\frac{m}{2}}\left|I_{\text {right }}[x+i][y+i]-I_{\text {left }}[x+i+d][y+j]\right|
$$

$m$ is the template size. $I_{\text {left }}$ and $I_{\text {right }}$ are the left and right images. For each pixel in the image, the neighborhoods are selected according to a square of a given size. To judge whether the feature points are the same points, a series of neighborhoods along the same row in another camera are compared with them. The judgment to determine whether they are the same points is the smallest value of $J$.

\section{MEASUREMENT PROCESSES AND IMAGE CORRECTION}

Figure 3 shows the measurement process of binocular stereo vision system in this paper. 


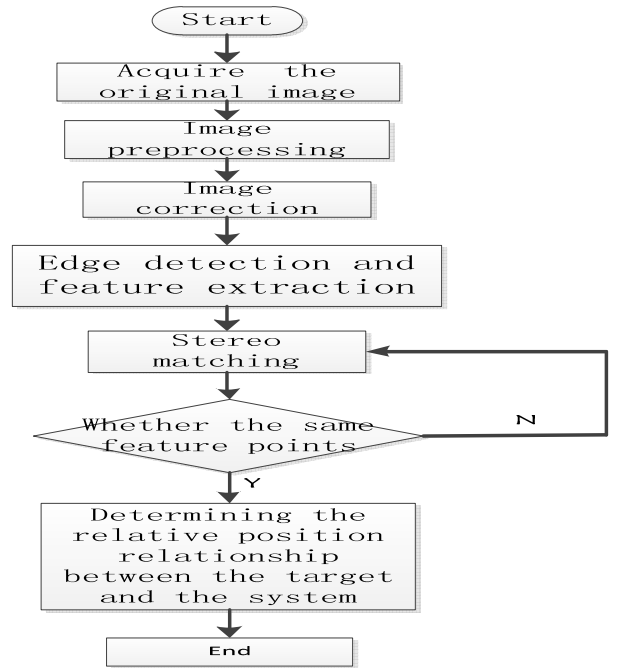

Figure 3. Measurement processes

Firstly, the cameras grab images. Low-pass filter for image preprocessing is used to eliminate image noise. Transmission transform is followed to correct system lens distortion. Then to extract the edges and features information of the target object uses sobel-edge detection algorithm. Finally the left and right cameras do stereo matching based on the feature points' absolute related deviation sum. At last we can calculate the spatial position of the measured target.

Distortion is inherent in the camera. So it's necessary to do image correction for lens distortion correction for reducing the measurement error. Transmission transform method is used for image correction in this paper and shown in formula 6 .

$$
\left\{\begin{array}{l}
u=\frac{a x+b y+c}{g x+h y+1} \\
v=\frac{d x+e y+f}{g x+h y+1}
\end{array}\right.
$$

$(\mathrm{x}, \mathrm{y})$ are pixel coordinates in reference image coordinate system. $(\mathrm{u}, \mathrm{v})$ are the corresponding pixel coordinates of the transformed image. a, b, c, d, e, f, g and h represent the transform coefficients. Transform coefficients are inner parameters of the camera which determined by using the calibration template to calibrate the cameras. For a given point in space, the transform coefficients' formula is shown in formula 7.

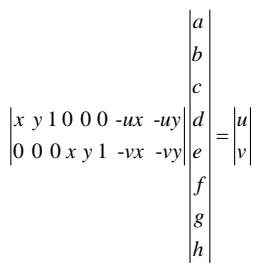

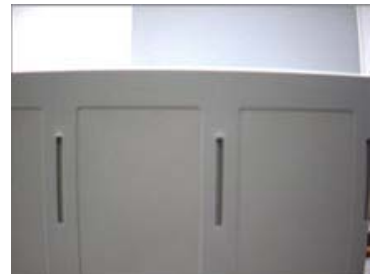

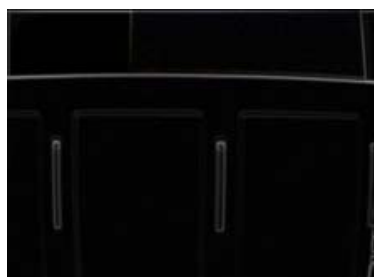

$\begin{array}{ll}\text { (a) Original image } & \text { (b) Edge extraction results of original image }\end{array}$

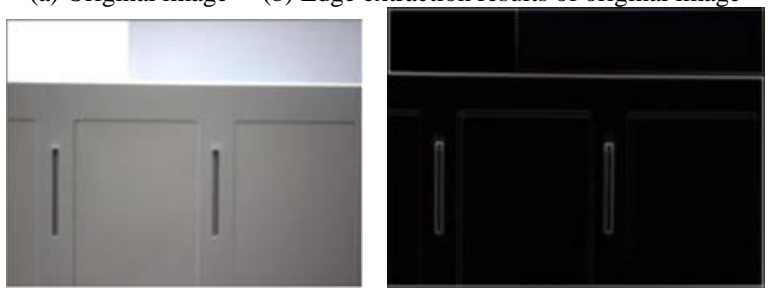

(c) Corrected image (d) Edge extraction results of corrected image

Figure 4. Image before and after correction

Figure 4 are the comparing results before and after correction. Figure 4 (a), (b) is the original image and its edge. Figure 4 (c), (d) is the image and its edge after correction. By comparing the images, it shows apparently the distorted edge of the original image due to distortion has been corrected to straight line.

The stereo vision system first extracts the original stereoscopic image. Then extracting feature information of the edge and target object after correction, finally to determine whether they are the same points and the relative position relationship between the target object and the measurement system through stereo matching.

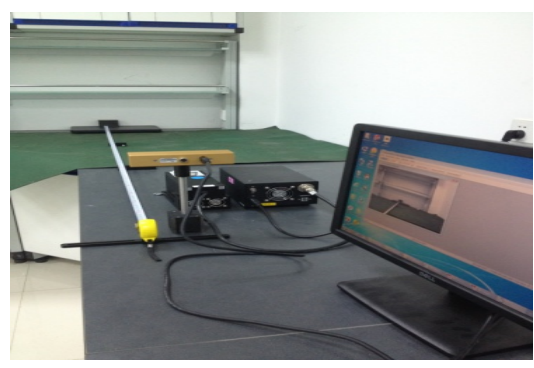

Figure 5. Environment and equipment

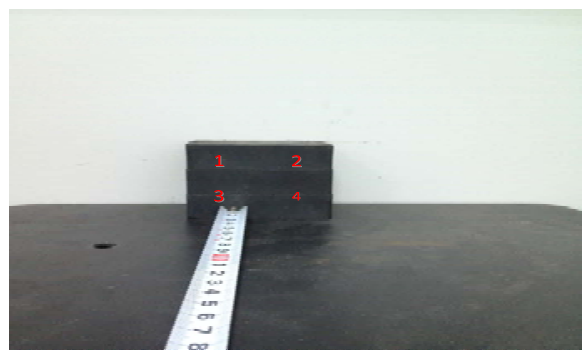

Figure 6. Target object and feature points

\section{RESULTS OF EXPERIMENTS}

Experiments have done in order to verify the feasibility and accuracy of the method proposed in this paper. Figure 5 shows environment and apparatus of experiment. The 
distance between the object and the vision measurement system is measured. Four feature points are randomly selected and their three-dimensional coordinates are acquired. Then calculate the average distance between feature points and the center of the vision. The result getting by steel tape is as a basis for judging the accuracy of the feature point distance measurement.

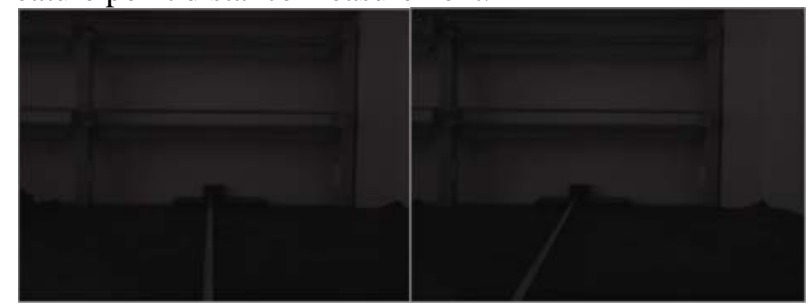

(a) Left image without structured light (b) Right image without structured
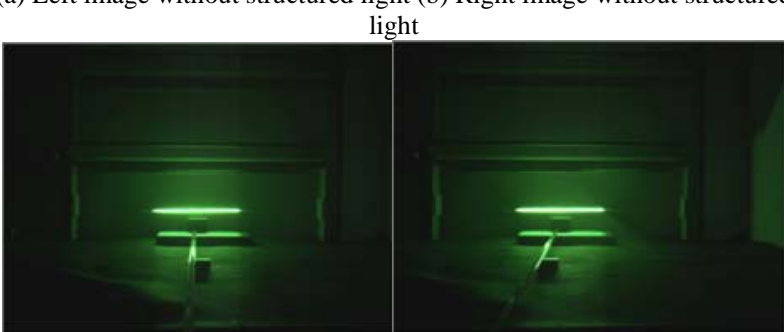

(c) Left image with structured light (d) Right image with structured light

Figure 7. Images before and after using structured light illumination

Figure 7 shows the image taken by cameras before and after using structured light. The measured target edge can be clearly distinguished after adding structured light illumination. It can be seen the measured object can be clearly distinguished edges after adding structured light illumination. The parallax image of view field processed by the method in this paper is shown in figure 8. The result of the four points' distance measurement is shown in Table I.

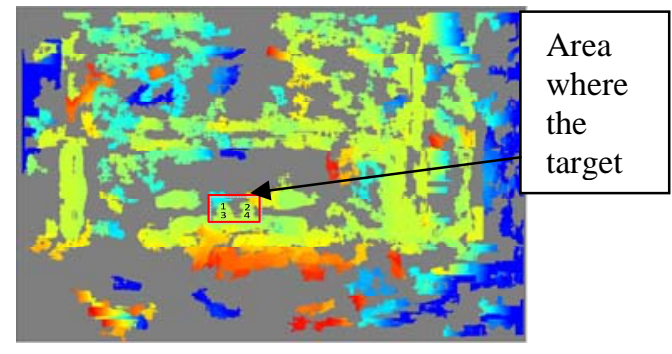

Figure 8. Parallax image of system
TABLE I. THE RESULT OF THE MEASUREMENT

\begin{tabular}{ccccc}
\hline No. & Measurements(m) & $\begin{array}{c}\text { Average } \\
\text { value(m) }\end{array}$ & $\begin{array}{c}\text { Standard } \\
\text { value(m) }\end{array}$ & Error(\%) \\
\hline 1 & 2.13 & & & \\
2 & 2.14 & 2.135 & 2.115 & 0.9 \\
3 & 2.12 & & & \\
4 & 2.15 & & & \\
\hline
\end{tabular}

Structured light is added effectively to reduce the impact of insufficient light and increase the success rate of matching through calculation. The measurement error is less than $1 \%$ which meets the measurement needs in insufficient illumination environment.

\section{CONCLUSIONS}

This paper presents a structured light stereo vision measurement system that can efficiently extract the measured target feature information in low-light environments. The method mentioned in this paper can effectively corrected lens distortion. The feature point matching method based on absolute related deviation sum can accurately achieve three-dimensional reconstruction. The method said by the paper is able to measure the measured target distance in low-light environment. The accuracy meets the requirement in generally underwater odometry and topography mapping.

\section{ACKNOWLEDGEMENTS}

The research work was supported by Science and Technology Department of Zhejiang Province under the No. 2013 C31071.

\section{REFERENCES}

[1] Huiwen Leng, Chunguang $\mathrm{Xu}$, Dingguo Xiao, etal. A method for measuring complicated deep-Hole profile using Line-StructuredLight sensor. Transactions of Beijing Institute of Technology, 33(2),pp.139-143,February 2013.

[2] Jun Zhao, Ji Zhao, Lei Zhang. Image Processing and Feature Extraction for Structured Light Images of Welded Seam. Journal of xi'an jiaotong university, 47(1),pp.114-119, January,2013.

[3] Yinqiao Cai, Xiaohua Tong, Rong Shu. Fast 3D Imaging with Time-offlight LaserScanner Based on Linear Array Scanning. Journal of tongji university(natural.science), 39(7), pp.1062-1067,July 2011.

[4] Jian Wang, XiangjunWang, Feng Liu. Modeling of binocular stereo vision for remote coordinate measurement and fast calibration. Optics and Lasers in Engineering ,pp.269-274,2014.

[5] Haitao lang, Liren Liu, Qingguo Yang. A Three-Dimensional Imaging Method Based on the Principle of Coded Aperture Imaging. Acta Optica Sinica,26(1),pp.34-38,January 2006.

[6] Hongqiang Huang, Huajun Feng, Zhihai xu et al. 3-D Imaging based on color-encoded structure light. Journal of Zhejiang University(Engineering Science),35(6),pp.588-591, November 2011.

[7] Jing Wei, Chengyuan Song, Xuqiang Li et al. Imaging by Single-Slit Streak Tube Laser Lidar,35(4),pp.496-500,April 2008.

[8] Cheng Cao, Xuyun Nie, Zheng Chen. New Auto Identification Technology on Paper Currency Using Pseudo Binocular Stereo Imaging. Computer Science,39(1),pp.19-22, January 2012 